Ibn Al-Haitham Jour. for Pure \& Appl. Sci. 33 (4) 2020

Ibn Al Haitham J ournal for Pure and Applied Science

J ournal homepage: http://jih.uobaghdad.edu.iq/index.php/j/index

\title{
Comparison Among Three Estimation Methods to Estimate Cascade Reliability Model (2+1) Based On Inverted Exponential Distribution
}

\author{
Sairan Hamza Raheem \\ University of Garmian, College of Computing and Information Technology, IT. Department \\ sairan.hamza@garmian.edu.krd
}

Article history: Received 13 January 2020, Accepted 12 February 2020, Published in October 2020

Doi: 10.30526/33.4.2512

\begin{abstract}
In this paper, we are mainly concerned with estimating cascade reliability model $(2+1)$ based on inverted exponential distribution and comparing among the estimation methods that are used. The maximum likelihood estimator and uniformly minimum variance unbiased estimators are used to get the parameters of the strengths $X_{k}$ and the stress $Y_{k} ; \mathrm{k}=1,2,3$ respectively then, by using the unbiased estimators, we propose Preliminary test single stage shrinkage (PTSSS) estimator when a prior knowledge is available for the scale parameter as initial value due past experiences. The Mean Squared Error [MSE] for the proposed estimator is derived to compare among the methods. Numerical results about conduct of the considered estimator are discussed including the study of mentioned expressions. The numerical results are exhibited and put it in tables.
\end{abstract}

Keyword: Inverted exponential distribution, Maximum Likelihood method, Uniformly Minimum Variance Unbiased method, Single Stage Shrinkage Estimator, Mean Squared Error and Cascade Reliability Model (2+1).

\section{Introduction}

An $n$ - cascade system is defined as a special type of standby system with $n$ components and it could be considered as a kind of stress-strength model. The reliability system of a cascade model can be described by a function of parameters of the identical and independent distributions with strength $(\mathrm{X})$ and stress $(\mathrm{Y})$ and the attenuation factor $(\mathrm{K})$ in other words, the stresses on subsequent components are attenuated by a factor ' $k$ ', called attenuation factor that is generally assumed to be a constant for all the components or to be a parameter having different fixed values for different components, or it may be simply a random variable. This 
system was first proposed and studied by Pandit and Sriwastav (1975).Rekha and Chechu Raju (1999) presented a closed form solution of stress attenuated reliability function for ncascade system with exponential stress and standby strengths following Rayleigh and exponential distributions. Sundar (2012)has done a case study of cascade reliability with Rayleigh distribution. Devi (2016) studied Cascade System Reliability with Stress and Strength Follow Lindley Distribution. Dong and Cui studied System Reliability under Cascading Failure Models. Doloi and Gogoi (2017) presented a cascade reliability model for exponential and Lindley distributions. Reddy(2016) studied Cascade and System Reliability for Exponential Distributions. In (2019) Kanaparthi et el studied Cascade and System Reliability for the New Rayleigh-Pareto Distribution

The aim of this study is to compare among three estimation methods (MLE ,UMVUE and PTSSs) in estimate the cascade reliability model $(2+1)$ based on inverted exponential distribution (IE) with unknown scale parameter. For this purpose, we use Thamson shrinkage technique through the equation below:

$\tilde{\alpha}=\emptyset(\hat{\alpha})\left(\hat{\alpha}-\alpha_{0}\right)+\alpha_{0}$

Where $\alpha_{0}$ is a prior knowledge(estimation) about the parameter $\alpha$ and $0 \leq \emptyset(\hat{\alpha}) \leq 1$ is a shrinkage weight factor to assign the degree of belief in $\alpha_{0}$; also $\hat{\alpha}$ is the classical estimator of $\alpha$ (MLE or UMVUE). Several authors have studied the estimator defined in (1) for a special distribution for different parameters and suitable regions (R) as well as for estimate the parameters of linear regression model. See [13-16].

\section{Statistical Model}

In life distribution, if a random variable $X$ following an exponential distribution then the variable $Y=\frac{1}{X}$ has an inverted exponential distribution (IE) . The Invers Exponential Distribution $\operatorname{IE}(\alpha)$ has the following probability density function p.d.f and cumulative distribution function c.d.f. for $x>0$ :

$f(x ; \alpha)=\frac{\alpha}{x^{2}} \exp \left(\frac{-\alpha}{x}\right) \quad ; \alpha>0$

$F(x ; \alpha)=\exp \left(\frac{-\alpha}{x}\right) ; \alpha>0$

Killer and Kamath (1982) defined and studied IE for the first time. Lin et al (1989) descripted IE distribution as a lifetime model. It can be seen that IE distribution was widely used in the analytic study specially in engineering, biology and medicine fields Oguntunde et el(2017). Bayes estimators of the parameter and reliability function of the IE distribution were obtained by Singh et al(2013). Oguntunde et al. (2014) proposed exponentiated GIE (EGIE) distribution. Singh et al (2015) estimated the stress strength reliability parameter of IE distribution. Fatima and Ahmad (2018) used a Bayesian Approximation Techniques of Inverse Exponential Distribution with Applications in Engineering.

For cascade model $(2+1)$, assume that $X_{1}, X_{2}$ and $X_{3}$ are the strengths with two fundamental components and one standby component is i.i.d with p.d.f follows $\operatorname{IED}(\alpha)$ with scale parameter $\alpha_{i} ; i=1,2,3$, when activated faces the stresses random variables $Y_{1}, Y_{2}$ and $Y_{3}$ are imposed on the strengths components and followed $\operatorname{IED}(\beta)$ with scale parameter $\beta_{j} ; j=$ $1,2,3$. In cascade system, after every failure the stress gets modified by attenuation factor $(\mathrm{k})$ such that: $Y_{2}=k Y_{1}, Y_{3}=k Y_{2}=k^{2} Y_{1}, \ldots, Y_{i}=k^{i-1} Y_{i} ; k>1$ 
And we suppose a factor (m) to modified the strength such that: $X_{2}=m X_{1}, X_{3}=m X_{2}=$ $m^{2} X_{1}, \ldots, X_{i}$

$$
=m^{i-1} X_{1} ; \mathrm{m}>0
$$

The real reliability function for the $(2+1)$ cascade model is given by:

$$
\begin{aligned}
& R=p\left[X_{1} \geq Y_{1}, X_{2} \geq Y_{3}\right]+p\left[X_{1}<Y_{1}, X_{2} \geq Y_{2}, X_{3} \geq Y_{3}\right]+p\left[X_{1} \geq\right. \\
& \left.Y_{1}, X_{2}<Y_{2}, X_{3} \geq Y_{3}\right] \\
& R=R_{1}+R_{2}+R_{3}
\end{aligned}
$$

$$
\begin{aligned}
R_{1}= & p\left[X_{1} \geq Y_{1}, X_{2} \geq Y_{2}\right] \\
= & \int_{0}^{\infty}\left[\bar{F}_{x_{1}}\left(y_{1}\right)\right] g\left(y_{1}\right) d y_{1} \int_{0}^{\infty}\left[\bar{F}_{x_{2}}\left(y_{2}\right)\right] g\left(y_{2}\right) d y_{2} \\
= & \int_{0}^{\infty}\left[1-\exp \left(\frac{-\alpha_{1}}{y_{1}}\right)\right] \frac{\beta_{1}}{y_{1}{ }^{2}} \exp \left(\frac{-\beta_{1}}{y_{1}}\right) d y_{1} \int_{0}^{\infty}\left[1-\exp \left(\frac{-\alpha_{2}}{y_{2}}\right)\right] \frac{\beta_{2}}{y_{2}{ }^{2}} \exp \left(\frac{-\beta_{2}}{y_{2}}\right) d y_{2} \\
& \text { So we get } \\
& R_{1}=\left[\frac{\alpha_{1}}{\alpha_{1}+\beta_{1}}\right]\left[\frac{\alpha_{2}}{\alpha_{2}+\beta_{2}}\right] \\
R_{2}= & p\left[X_{1}<Y_{1}, X_{2} \geq Y_{2}, X_{3} \geq Y_{3}\right] \\
= & p\left[X_{1}<Y_{1}, m X_{1} \geq k Y_{1}\right] p\left[X_{2} \geq Y_{2}\right] p\left[X_{2} \geq Y_{2}\right]
\end{aligned}
$$

where

$$
\begin{aligned}
p\left[X_{1}<Y_{1}, m X_{1} \geq k Y_{1}\right] & =\int_{0}^{\infty}\left[F_{x_{1}}\left(y_{1}\right)\right]\left[\bar{F}_{x_{1}}\left(\frac{k}{m} y_{1}\right)\right] g\left(y_{1}\right) d y_{1} \\
& =\int_{0}^{\infty} \exp \left(\frac{-\alpha_{1}}{y_{1}}\right)\left[1-\exp \left(\frac{-\alpha_{1}}{\frac{k}{m} y_{1}}\right)\right] \frac{\beta_{1}}{y_{1}{ }^{2}} \exp \left(\frac{-\beta_{1}}{y_{1}}\right)
\end{aligned}
$$

Then $p\left[X_{1}<Y_{1}, m X_{1} \geq k Y_{1}\right]=\frac{\left(\frac{k}{m} \alpha_{1}\right) \beta_{1}}{\left(\frac{k}{m} \alpha_{1}+\beta_{1}\right)\left(\alpha_{1}+\frac{k}{m} \alpha_{1}+\beta_{1}\right)}$

And

$p\left[X_{2} \geq Y_{2}\right]=\int_{0}^{\infty}\left[\bar{F}_{x_{2}}\left(y_{2}\right)\right] g\left(y_{2}\right) d y_{2}=\frac{\alpha_{2}}{\alpha_{2}+\beta_{2}}$

So

$R_{2}=\left[\frac{\left(\frac{k}{m} \alpha_{1}\right) \beta_{1}}{\left(\frac{k}{m} \alpha_{1}+\beta_{1}\right)\left(\alpha_{1}+\frac{k}{m} \alpha_{1}+\beta_{1}\right)}\right]\left(\frac{\alpha_{2}}{\alpha_{2}+\beta_{2}}\right)$

By the same way finding $\mathrm{R}_{3}$

$$
\begin{aligned}
\mathrm{R}_{3}= & p\left[X_{1} \geq Y_{1}, X_{2}<Y_{2}, X_{3} \geq Y_{3}\right] \\
= & {\left[\frac{\frac{k}{m} \alpha_{2} \beta_{2}}{\left(\frac{k}{m} \alpha_{2}+\beta_{2}\right)\left(\alpha_{2}+\frac{k}{m} \alpha_{2}+\beta_{2}\right)}\right]\left(\frac{\alpha_{1}}{\alpha_{1}+\beta_{1}}\right) }
\end{aligned}
$$

Substitution (6), (11) and (13) in (4)

$$
\begin{gathered}
R=\left[\frac{\alpha_{1}}{\alpha_{1}+\beta_{1}}\right]\left[\frac{\alpha_{2}}{\alpha_{2}+\beta_{2}}\right]+\left[\frac{\frac{k}{m} \alpha_{1} \beta_{1}}{\left(\frac{k}{m} \alpha_{1}+\beta_{1}\right)\left(\alpha_{1}+\frac{k}{m} \alpha_{1}+\beta_{1}\right)}\right]\left(\frac{\alpha_{2}}{\alpha_{2}+\beta_{2}}\right) \\
+\left[\frac{\frac{k}{m} \alpha_{2} \beta_{2}}{\left(\frac{k}{m} \alpha_{2}+\beta_{2}\right)\left(\alpha_{2}+\frac{k}{m} \alpha_{2}+\beta_{2}\right)}\right]\left(\frac{\alpha_{1}}{\alpha_{1}+\beta_{1}}\right)
\end{gathered}
$$




\section{Maximum Likelihood Estimation (MLE)}

The Maximum likelihood method is an important and commonly, since it contained properties for good estimate. Suppose $X_{i} ; i=1,2,3$ strength random sample follows $\operatorname{IED}(\alpha)$ with the sample size $n$.The likelihood function is given by; $l=L\left(x_{1}, x_{2}, \ldots, x_{n} ; \alpha\right)$

$$
\begin{aligned}
& =\prod_{i=1}^{n} f\left(x_{i}\right)=\prod_{i=1}^{n} \frac{\alpha}{x_{i}{ }^{2}} \exp \left(\frac{-\alpha}{x_{i}}\right) \\
& =\alpha^{n} \prod_{i=1}^{n} \frac{1}{x_{i}{ }^{2}} \exp \left(\frac{-\alpha}{\sum_{i=1}^{n} x_{i}}\right)
\end{aligned}
$$

Taking logarithm of (16) and then differentiating the result partially with respect to $\alpha$ :

$\frac{\partial \ln L}{\alpha}=\frac{n}{\alpha}-\sum_{i=1}^{n} \frac{1}{x_{i}}$

Equalizing (17) to zero to get the estimated scale parameter of IED

$\hat{\alpha}_{M L E}=\frac{n}{\sum_{i=1}^{n} \frac{1}{x_{i}}}$

Hence, for strength random samples, $X_{1_{i_{1}}} \sim \operatorname{IE}\left(\alpha_{1}\right) ; i_{1}=1,2, \ldots, n_{1}, X_{2_{i_{2}}} \sim \operatorname{IE}\left(\alpha_{2}\right) ; i_{2}=$ $1,2, \ldots, n_{2}$ and $X_{3_{i_{3}}} \sim \operatorname{IE}\left(\alpha_{3}\right) ; i_{3}=1,2, \ldots, n_{3}$ with samples size $n_{1}, n_{2}$ and $n_{3}$ respectively MLE for unknown parameters $\alpha_{1}, \alpha_{2}$ and $\alpha_{3}$ is :

$\hat{\alpha}_{\Lambda(M L E)}=\frac{n_{\Lambda}}{T_{\Lambda}} ; T_{\Lambda}=\sum_{i_{\Lambda}=1}^{n_{\Lambda}} \frac{1}{x_{\Lambda_{i_{\Lambda}}}} ; \Lambda=1,2,3$

By the same way for the stress random variables $Y_{1_{1}} \sim \operatorname{IE}\left(\beta_{1}\right) ; j_{1}=1,2, \ldots, m_{1}$, $Y_{2_{j_{2}}} \sim I E\left(\beta_{2}\right) ; j_{2}=1,2, \ldots, m_{2}$ and $Y_{3_{j_{3}}} \sim \operatorname{IE}\left(\beta_{3}\right) ; j_{3}=1,2, \ldots, m_{3}$ with samples size $m_{1}$ , $m_{2}$ and $m_{3}$ respectively, the MLE estimator for unknown parameters $\beta_{1}, \beta_{2}$ and $\beta_{3}$ will be as follows:

$\hat{\beta}_{\Lambda(M L E)}=\frac{m_{\Lambda}}{H_{\Lambda}} ; H_{\Lambda}=\sum_{j_{\Lambda}=1}^{m_{\Lambda}} \frac{1}{y_{\Lambda_{j_{\Lambda}}}}, \quad \Lambda=1,2,3$

Substitution (19) and (20) in (15) the MLE for Cascade reliability, invariability will be as:

$$
\begin{aligned}
\widehat{R}_{M L E}=\left[\frac{\widehat{\alpha}_{1(M L E)}}{\widehat{\alpha}_{1(M L E)}+\widehat{\beta}_{1(M L E)}}\right]\left[\frac{\widehat{\alpha}_{2(M L E)}}{\widehat{\alpha}_{2(M L E)}+\widehat{\beta}_{2(M L E)}}\right] \\
+\left[\frac{\frac{k}{m} \widehat{\alpha}_{1(M L E)} \widehat{\beta}_{1(M L E)}}{\left(\frac{k}{m} \widehat{\alpha}_{1(M L E)}+\widehat{\beta}_{1(M L E)}\right)\left(\widehat{\alpha}_{1(M L E)}+\frac{k}{m} \widehat{\alpha}_{1(M L E)}+\widehat{\beta}_{1(M L E)}\right)}\right]\left(\frac{\widehat{\alpha}_{2(M L E)}}{\widehat{\alpha}_{2(M L E)}+\widehat{\beta}_{2(M L E)}}\right) \\
+\left[\frac{\frac{k}{m} \widehat{\alpha}_{2(M L E)} \widehat{\beta}_{2(M L E)}}{\left(\frac{k}{m} \widehat{\alpha}_{2(M L E)}+\widehat{\beta}_{2(M L E)}\right)\left(\widehat{\alpha}_{2(M L E)}+\frac{k}{m} \widehat{\alpha}_{2(M L E)}+\widehat{\beta}_{2(M L E)}\right)}\right]\left(\frac{\widehat{\alpha}_{1(M L E)}}{\widehat{\alpha}_{1(M L E)}+\widehat{\beta}_{1(M L E)}}\right)
\end{aligned}
$$

\section{Uniformly Minimum Variance Unbiased Estimators}

The UMVUE method depends on minimizing the mean square error among unbiased estimators. The unbiased estimator $\widehat{\alpha}$ of $\alpha$ is called (UMVUE) if and only if $\operatorname{Var}(\widehat{\alpha}) \leq$ $\operatorname{Var}\left(\widehat{\alpha}_{u b}\right)$ for any $x \in X$ and any other unbiased estimator of $\alpha$, see Devor and Berk (2012). We could find the UMVU of the scale parameters $\alpha_{\Lambda}$, and $\beta_{\Lambda} ; \Lambda=1,2,3$ of the stress $X_{\Lambda_{i}} ; i_{\Lambda}=$ $1,2, \ldots, n_{\Lambda}$ and strength $Y_{\Lambda_{j_{\Lambda}}} j_{\Lambda}=1,2, \ldots, m_{\Lambda}$; of cascade reliability model with IED that belongs to exponential family densities function as it shown below :

$Q(x ; \sigma)=a(\sigma) b(x) \exp \left(\sum_{j=1}^{m} \rho_{j}(\sigma) r_{j}(x)\right)$, where $a(\sigma), b(x)>o$, and $\sigma \sigma_{1}, \sigma_{2}, \ldots, \sigma_{r}$ with $\gamma_{j}<\sigma_{j}<\delta_{j}$ and each of $\sigma, \gamma_{j}$ and $\delta_{j}$ are constant . 
Let $a(\sigma)=\alpha_{1} ; b(x)=1 / x^{2} \quad ; \rho_{j}(\sigma)=-\alpha_{1} ; r_{j}(x)=\frac{1}{x}$

Thus, $T_{\Lambda}$ is a complete sufficient statistic for $\left(\alpha_{\Lambda}\right)$ for $\Lambda=1,2,3$.

To find the distribution of $T_{1}=\sum_{i=1}^{n_{1}} \frac{1}{x_{1_{1}}}$, suppose $Z_{1}=\frac{1}{X}$ consequently, $X=\frac{1}{Z_{1}}$

$p\left(z_{1}\right)=f\left(X=\frac{1}{z_{1}}\right) \frac{d x}{d z_{1}}$

Substitute (2) in (22)

$p\left(z_{1}\right)=\alpha_{1} \exp \left(-\alpha_{1} z_{1}\right)$

Thus $Z_{1} \sim \operatorname{Exp}\left(\alpha_{1}\right)$, and $T_{1} \sim \Gamma\left(n_{1}, \alpha_{1}\right)$

$U\left(t_{1}\right)=\frac{\alpha_{1}^{n_{1}}}{\Gamma\left(n_{1}\right)} t^{n_{1}-1} \cdot \exp \left(-\alpha_{1} t_{1}\right) \quad ; t_{1}>0, \alpha_{1}>0, n_{1}>0$

then $E\left(\frac{1}{T_{1}}\right)=\frac{\alpha_{1}}{n_{1}-1}$

So the unbiased estimator of $\left(\alpha_{1}\right)$ is $\left(\frac{n_{1}-1}{T_{1}}\right)$, therefore according to Lehmann-Scheffe theorem the (UMVUE) of $\left(\alpha_{1}\right)$ is

$\hat{\alpha}_{1(U M V U)}=\frac{n_{1}-1}{T_{1}}$

Hence

$\hat{\alpha}_{\Lambda(U M V U)}=\frac{n_{\Lambda}-1}{T_{\Lambda}} ; T_{\Lambda}=\sum_{i_{\Lambda}=1}^{n_{\Lambda}} \frac{1}{x_{\Lambda_{i_{\Lambda}}}}, k 1,2,3$

By the same way, we can obtain (UMVUE) of $\left(\beta_{K}\right)$ as below:

$\hat{\beta}_{\Lambda(U M V U)}=\frac{m_{\Lambda}-1}{H_{\Lambda}} \quad ; H_{\Lambda}=\sum_{j_{\Lambda}=1}^{m_{\Lambda}} \frac{1}{y_{\Lambda_{j_{\Lambda}}}} \quad, \Lambda=1,2,3$

Substituting (25), (26) in (15) to obtain UMVU estimator for cascade reliability model of IED as the following:

$$
\begin{aligned}
& \widehat{R}_{(U M V U)}\left[\frac{\widehat{\alpha}_{1(U M V U)}}{\widehat{\alpha}_{1(U M V U)}+\widehat{\beta}_{1(U M V U)}}\right]\left[\frac{\widehat{\alpha}_{2(U M V U)}}{\widehat{\alpha}_{2(U M V U)}+\widehat{\beta}_{2(U M V U)}}\right] \\
& +\left[\frac{\frac{k}{m} \widehat{\alpha}_{1(U M V U)} \widehat{\beta}_{1(U M V U)}}{\left(\frac{m}{k} \widehat{\alpha}_{1(U M V U)}+\widehat{\beta}_{1(U M V U)}\right)\left(\widehat{\alpha}_{1(U M V U)}+\frac{m}{k} \widehat{\alpha}_{1(U M V U)}+\widehat{\beta}_{1(U M V U)}\right)}\right]\left(\frac{\widehat{\alpha}_{2(U M V U)}}{\widehat{\alpha}_{2(U M V U)}+\widehat{\beta}_{2(U M V U)}}\right) \\
& +\left[\frac{\frac{k}{m} \widehat{\alpha}_{2(U M V U)} \widehat{\beta}_{2(U M V U)}}{\left(\frac{k}{m} \widehat{\alpha}_{2(U M V U)}+\widehat{\beta}_{2(U M V U)}\right)\left(\widehat{\alpha}_{2(U M V U)}+\frac{k}{m} \widehat{\alpha}_{2(U M V U)}+\widehat{\beta}_{2(U M V U)}\right)}\right]\left(\frac{\widehat{\alpha}_{1(U M V U)}}{\widehat{\alpha}_{1(U M V U)}+\widehat{\beta}_{1(U M V U)}}\right)
\end{aligned}
$$

\section{Preliminary Test Single Shrinkage Estimator (PTSSSE).}

In this section, we use (1) to estimate the cascade reliability parameters $\alpha_{\Lambda}, \beta_{\Lambda} ; \Lambda=1,2,3$ of $X_{\Lambda} \sim I E D$ and $Y_{\Lambda} \sim I E D$ respectively. First in order to find PTSSSE of $\alpha_{\Lambda}$ choose $\emptyset\left(\hat{\alpha}_{\Lambda}\right)$ as follows:

$$
\emptyset\left(\hat{\alpha}_{\Lambda}\right)= \begin{cases}\vartheta_{1}\left(\hat{\alpha}_{\Lambda}\right) & \text { if } \hat{\alpha}_{\Lambda} \in R_{p t_{1}} \\ \vartheta_{2}\left(\hat{\alpha}_{\Lambda}\right) & \text { if } \hat{\alpha}_{\Lambda} \notin R_{p t_{1}}\end{cases}
$$

Where $R_{p t_{1}}$ is a preliminary test region of acceptances of size $\left(\lambda_{1}\right)$ for testing hypotheses $H_{0}: \alpha_{\Lambda}=\alpha_{\Lambda_{0}}$ vs. $H_{A}: \alpha_{\Lambda} \neq \alpha_{\Lambda_{0}}$ using the test statistic $\tau_{1}=\frac{\left(n_{\Lambda}-1\right) \widehat{\alpha}_{\Lambda}}{\alpha_{\Lambda_{0}}} ; \hat{\alpha}_{\Lambda}=\hat{\alpha}_{\Lambda(U M V U)}$ thus, by rewriting equation( 1$)$ as below:

$\tilde{\alpha}_{1}= \begin{cases}\vartheta_{1}\left(\hat{\alpha}_{\Lambda}\right)\left(\hat{\alpha}_{\Lambda}-\alpha_{\Lambda_{0}}\right)+\alpha_{\Lambda_{0}} & \text {, if } \hat{\alpha}_{\Lambda} \in R_{p t_{1}} \\ \vartheta_{2}\left(\hat{\alpha}_{\Lambda}\right)\left(\hat{\alpha}_{\Lambda}-\alpha_{\Lambda_{0}}\right)+\alpha_{\Lambda_{0}} & \text {, if } \hat{\alpha}_{\Lambda} \notin R_{p t_{1}}\end{cases}$

Where $\vartheta_{r}\left(\hat{\alpha}_{\Lambda}\right) ; r=1,2$ represents the shrinkage weight factor which may be a function of $\hat{\alpha}_{\Lambda}$ or may be constants with the condition $0 \leq \vartheta_{r}\left(\hat{\alpha}_{\Lambda}\right) \leq 1$. Using the form (29), we proposed 
two preliminary test single stage shrinkage estimators $\tilde{\alpha}_{s s s}$ when a prior knowledge $\alpha_{\Lambda_{0}}$ is available for $\alpha_{\Lambda}$

$\tilde{\alpha}_{s s S_{\Lambda_{1}}}= \begin{cases}\alpha_{\Lambda_{0}} & \text {, if } \hat{\alpha}_{\Lambda} \in R_{p t_{1}} \\ \hat{\alpha}_{\Lambda} & \text {, if } \hat{\alpha}_{\Lambda} \notin R_{p t_{1}}\end{cases}$

Then $\vartheta_{1}\left(\hat{\alpha}_{\Lambda}\right)=0$ and $\vartheta_{2}\left(\hat{\alpha}_{\Lambda}\right)=1$ in $(28)$

And

$\tilde{\alpha}_{s s S_{\Lambda_{2}}}= \begin{cases}\alpha_{\Lambda_{0}} & \text { if } \hat{\alpha}_{\Lambda} \in R_{p t_{1}} \\ c_{1}\left(\hat{\alpha}_{\Lambda}-\alpha_{\Lambda_{0}}\right) & \text {, if } \hat{\alpha}_{\Lambda} \notin R_{p t_{1}}\end{cases}$

Thus $\vartheta_{1}\left(\hat{\alpha}_{\Lambda}\right)=0$ and $\vartheta_{2}\left(\hat{\alpha}_{\Lambda}\right)=c_{1}$ in (28) while:

$R_{p t_{1}}=\left(a_{1}, a_{2}\right) ; \quad a_{1}=\frac{\alpha_{\Lambda_{0}}}{2 n_{\Lambda}} X_{1_{1-\lambda / 2,2 n_{\Lambda}}^{2}}$

And $a_{2}=\frac{\alpha_{\Lambda_{0}}}{2 n_{\Lambda}} X_{1 / 2,2 n_{\Lambda}}^{2}$

where $X_{1_{1-\lambda / 2,2 n_{\Lambda}}^{2}}, X_{1_{\lambda / 2,2 n_{\Lambda}}^{2}}$ are respectively the lower and upper $100(\lambda / 2)$ precedential point of chi-square distribution with $\left(2 n_{\Lambda}\right)$ degree of freedom.

Similarly for parameters $\beta_{\Lambda} ; \Lambda=1,2,3$ of stress random variables $Y_{\Lambda} \sim \operatorname{IED}$ choose $\emptyset_{2}\left(\hat{\beta}_{\Lambda}\right)$ as below:

$\emptyset_{2}\left(\hat{\beta}_{\Lambda}\right)= \begin{cases}\Theta_{1}\left(\hat{\beta}_{\Lambda}\right) & \text { if } \hat{\beta}_{\Lambda} \in R_{p t_{2}} \\ \Theta_{2}\left(\hat{\beta}_{\Lambda}\right) & \text { if } \hat{\beta}_{\Lambda} \notin R_{p t_{2}}\end{cases}$

Where $R_{p t_{2}}$ is a preliminary test region of acceptances of size $\left(\lambda_{2}\right)$ for testing hypotheses $\mathrm{њ}_{0}: \beta_{\Lambda}=\beta_{\Lambda_{0}}$ vs. $\biguplus_{A}: \beta_{\Lambda} \neq \beta_{\Lambda_{0}}$ using the test statistic $\tau_{2}=\frac{\left(m_{\Lambda}-1\right) \widehat{\beta}_{\Lambda}}{\beta_{\Lambda_{0}}} ; \hat{\beta}_{\Lambda}=\hat{\beta}_{\Lambda(U M V U)}$ thus, we get:

$\tilde{\beta}_{\Lambda}= \begin{cases}\Theta_{1}\left(\hat{\beta}_{\Lambda}\right)\left(\hat{\beta}_{\Lambda}-\beta_{\Lambda_{0}}\right)+\beta_{\Lambda_{0}} & , \text { if } \hat{\beta}_{\Lambda} \in R_{p t_{2}} \\ \Theta_{2}\left(\hat{\beta}_{\Lambda}\right)\left(\hat{\beta}_{\Lambda}-\beta_{\Lambda_{0}}\right)+\beta_{\Lambda_{0}} & , \text { if } \hat{\beta}_{\Lambda} \notin R_{p t_{2}}\end{cases}$

Such that $0 \leq \Theta_{r}\left(\hat{\beta}_{\Lambda}\right) \leq 1, r=1,2$ and $\Theta_{r}\left(\hat{\beta}_{\Lambda}\right)$ represents as shrinkage weight factor which may be constants or functions of $\hat{\beta}_{\Lambda}$. We proposed two preliminary test single stage shrinkage estimators $\tilde{\beta}_{\text {sss }}$ to estimate the reliability parameters when a prior knowledge $\beta_{\Lambda_{0}}$ is available for $\beta_{\Lambda}$

$\tilde{\beta}_{s s s_{\Lambda_{1}}}= \begin{cases}\beta_{\Lambda_{0}} & \text {, if } \hat{\beta}_{\Lambda} \in R_{p t_{2}} \\ \hat{\beta}_{\Lambda} & \text {, if } \hat{\beta}_{\Lambda} \notin R_{p t_{2}}\end{cases}$

Then $\Theta_{1}\left(\hat{\beta}_{\Lambda}\right)=0$ and $\Theta_{2}\left(\hat{\beta}_{\Lambda}\right)=1$ in $(28)$

And

$\tilde{\beta}_{s s s_{\Lambda_{2}}}= \begin{cases}\beta_{\Lambda_{0}} & , \text { if } \hat{\beta}_{\Lambda} \in R_{p t_{2}} \\ c_{2}\left(\hat{\beta}_{\Lambda}-\beta_{k_{0}}\right) & , \text { if } \hat{\beta}_{\Lambda} \notin R_{p t_{2}}\end{cases}$

Thus $\Theta_{1}\left(\hat{\beta}_{\Lambda}\right)=0$ and $\Theta_{2}\left(\hat{\beta}_{\Lambda}\right)=c_{2}$ in $(28)$

Such that $R_{p t_{2}}=\left(s_{1}, s_{2}\right)$ while $s_{1}=\frac{\beta_{\Lambda_{0}}}{2 m_{\Lambda}} Y_{\Lambda_{1-\lambda_{2} / 2,2 m_{\Lambda}}^{2}}$ and $s_{2}=\frac{\beta_{\Lambda_{0}}}{2 m_{\Lambda}} Y_{\Lambda_{2} / 2,2 m_{k}}^{2}$

$Y_{1_{1-\lambda_{2} / 2,2 m_{\Lambda}}}^{2}, Y_{1_{\lambda_{2} / 2,2 m_{\Lambda}}^{2}}^{2}$ are respectively the lower and upper $100\left(\lambda_{2} / 2\right)$ precedential point of chi-square distribution with $\left(2 m_{k}\right)$ degree of freedom.

Substituting (30),(31),(34) and (35) in (15) to obtain preliminary test single Shrinkage estimator for cascade reliability model $(2+1)$ of IED as the following 
Ibn Al-Haitham Jour. for Pure \& Appl. Sci. 33 (4) 2020

$$
\begin{aligned}
& \widehat{R}_{s S S}=\left[\frac{\widetilde{\alpha}_{1 s s s}}{\widetilde{\alpha}_{1 s s s}+\widetilde{\beta}_{1 s s s}}\right]\left[\frac{\widetilde{\alpha}_{2 s s s}}{\widetilde{\alpha}_{2 s s s}+\widetilde{\beta}_{2 s s s}}\right]+\left[\frac{\frac{k}{m} \widetilde{\alpha}_{1 s s s} \widetilde{\beta}_{1 s s s}}{\left(\frac{k}{m} \widetilde{\alpha}_{1 s s s}+\widetilde{\beta}_{1 s s s}\right)\left(\widetilde{\alpha}_{1 s s s}+\frac{k}{m} \widetilde{\alpha}_{1 s s s} \widetilde{\beta}_{1 s s s}\right)}\right]\left(\frac{\widetilde{\alpha}_{2 s s s}}{\widetilde{\alpha}_{1 s s s}+\widetilde{\beta}_{2 s s s}}\right) \\
& +\left[\frac{\frac{k}{m} \widetilde{\alpha}_{2 s s s} \widetilde{\beta}_{2 s s s}}{\left(\frac{k}{m} \widetilde{\alpha}_{2 s s S}+\widetilde{\beta}_{2 s s s}\right)\left(\widetilde{\alpha}_{2 s s s}+\frac{k}{m} \widetilde{\alpha}_{2 s s s}+\widehat{\beta}_{2(M L E)}\right)}\right]\left(\frac{\widetilde{\alpha}_{1 s s s}}{\widetilde{\alpha}_{1 S s s}+\widetilde{\beta}_{1 S s S}}\right)
\end{aligned}
$$

\section{Simulation Study}

In this section, we present some results based on Monte Carlo simulations to compare the performance of different methods: MLE, UMVUE and PTSSSE of Cascade Reliability Model(2+1) using different sample (20, 40, and 60). For this purpose the following steps of Monte Carlo simulation are used based on Mean Squared Errors criteria with 1000 replicates:

Step1: Generate random samples which follow the continuous uniform distribution defined on the interval $(0,1), \quad$ as $u_{i 1}, u_{i 2}, \ldots, u_{i j}$ and $w_{1}, w_{2}, \ldots, w_{m}$, for all $i=1,2, \ldots, k$ and $j=$ $1,2, \ldots, n_{i}$. respectively.,

Step2: Applying an inverse transformation approach to generate random variables follows IED as follows:

$F(x)=\exp \left(\frac{-\alpha}{x}\right): U i=\exp \left(\frac{-\alpha}{x}\right): x_{i}=\left[\alpha_{1} /-\ln (U i)\right]^{\frac{1}{2}}$

And, by the same method, we get:

$y_{i}=\left[\alpha_{2} /-\ln (V j)\right]^{\frac{1}{2}}$

Step3: Recall the R from equation (15).

Step4: find the cascade reliability R of the MLE using equation (21).

Step5: find the uniformly minimum variance unbiased method of $\mathrm{R}$ using equation (27).

Step6: Compute (PTSSSE) of cascade reliability using equations (36).

Step7: Calculate the MSE based on $(\mathrm{L}=1000)$ trials as follows:

$\operatorname{MSE}=\frac{1}{L} \sum_{i=1}^{L}\left(\hat{R}_{i}-R\right)^{2}$

\section{Results of Simulation}

In this section, the simulation results are used to determine the best outcome of the conceder estimation methods ( $M L E$, UMVUE, PTSSSE) of cascade reliability model $(2+1)$ estimator based on one parameter IED. In the cascade reliability model $(2+1)$ of estimate the system reliability $R=p\left[X_{1} \geq Y_{1}, X_{2} \geq Y_{3}\right]+p\left[X_{1}<Y_{1}, X_{2} \geq Y_{2}, X_{3} \geq Y_{3}\right]+$ $\left.\left.p\left[X_{1} \geq Y_{1}, X_{2}<Y_{2}, X_{3} \geq Y\right]_{3}\right)\right)$. The following tables of mean square error show, at most the orders rank of the estimators as follows: $\widehat{R}_{M L E}, \widehat{R}_{(U M V U)}$ and $\widehat{R}_{S S S}$ respectively, that means $\widehat{R}_{S S S}$ is the best than the others estimators. The following tables (1-9) will present the simulation results.

Table 1: $\alpha_{1}=1, \quad \alpha_{2}=1, \beta_{1}=1.5 \quad, \beta_{2}=1.5 \quad, \quad R=0.800000000$

\begin{tabular}{|l|l|l|l|l|l|}
\hline $\mathrm{k}$ & $\mathrm{m}$ & $(\mathrm{n} 1, \mathrm{n} 2, \mathrm{~m} 1, \mathrm{~m} 2)$ & $\mathrm{UM}$ & $\mathrm{PT}$ & $\mathrm{MLE}$ \\
\hline \multirow{2}{*}{1.5} & \multirow{2}{*}{0.2} & $(20,20,20,20)$ & 0.800040031839184 & 0.800000399098201 & 0.800055821748313 \\
\cline { 3 - 6 } & & $(60,60,20,40)$ & 0.800040032049879 & 0.800000862941454 & 0.800045359839790 \\
\hline 1.8 & \multirow{2}{*}{0.5} & $(40,20,60,60)$ & 0.800040032014180 & 0.7999999583412720 & 0.800053589528536 \\
\cline { 3 - 6 } & & $(20,40,60,20)$ & 0.800040031806876 & 0.7999999000261255 & 0.800019751832772 \\
\hline 1.3 & \multirow{2}{*}{0.7} & $(40,60,20,40)$ & 0.800040032055934 & 0.799999163925098 & 0.800033275056292 \\
\cline { 3 - 6 } & & $(60,20,40,60)$ & 0.800040032013649 & 0.800000092631102 & 0.800056080018052 \\
\hline \multirow{2}{*}{1.1} & \multirow{2}{*}{0.9} & $(40,40,40,40)$ & 0.800040032025629 & 0.8000000881956533 & 0.800050772085346 \\
\cline { 3 - 6 } & & $(60,60,60,60)$ & 0.800040032025629 & 0.7999999795970744 & 0.800040301978738 \\
\hline
\end{tabular}




\section{Ibn Al-Haitham Jour. for Pure \& Appl. Sci. 33 (4) 2020}

Table2: $M S E \alpha_{1}=1, \alpha_{2}=1, \beta_{1}=1.5, \beta_{2}=1.5$

\begin{tabular}{|l|l|l|l|l|l|}
\hline $\mathrm{k}$ & $\mathrm{m}$ & $(\mathrm{n} 1, \mathrm{n} 2, \mathrm{~m} 1, \mathrm{~m} 2)$ & $\mathrm{UM}$ & $\mathrm{PT}$ & MLE \\
\hline 1.5 & \multirow{2}{*}{0.2} & $(20,20,20,20)$ & 0.000000001602548 & 0.000000000868428 & 0.000000071936176 \\
\cline { 3 - 6 } & & $(60,60,20,40)$ & 0.000001602565018 & 0.000000456950252 & 0.000042789507494 \\
\hline 1.8 & \multirow{2}{*}{0.5} & $(40,20,60,60)$ & 0.000001602562159 & 0.000000397091475 & 0.000042894580602 \\
\cline { 3 - 6 } & & $(20,40,60,20)$ & 0.000000001602546 & 0.000000000960463 & 0.000000345959007 \\
\hline \multirow{2}{*}{1.3} & \multirow{2}{*}{0.7} & $(40,60,20,40)$ & 0.000001602565503 & 0.000000433528185 & 0.000032505999070 \\
\cline { 3 - 6 } & & $(60,20,40,60)$ & 0.000001602562117 & 0.000000382471540 & 0.000044937037854 \\
\hline 1.1 & \multirow{2}{*}{0.9} & $(40,40,40,40)$ & 0.000001602563075 & 0.000000389473349 & 0.000032823139814 \\
\cline { 3 - 6 } & & $(60,60,60,60)$ & 0.000001602563075 & 0.000000306936893 & 0.000014943488773 \\
\hline
\end{tabular}

Table 3: $\alpha_{1}=1.5, \alpha_{2}=1.5, \beta_{1}=2, \beta_{2}=2, R=0.7857142857$

\begin{tabular}{|l|l|l|l|l|l|}
\hline $\mathrm{k}$ & $\mathrm{m}$ & $(\mathrm{n} 1, \mathrm{n} 2, \mathrm{~m} 1, \mathrm{~m} 2)$ & $\mathrm{UM}$ & $\mathrm{PT}$ & MLE \\
\hline 1.5 & \multirow{2}{*}{0.2} & $(20,20,20,20)$ & 0.785734705446280 & 0.785714149871148 & 0.785720768248288 \\
\cline { 3 - 6 } & & $(60,60,20,40)$ & 0.785734705585085 & 0.785713939601543 & 0.785737729080948 \\
\hline \multirow{2}{*}{1.8} & \multirow{2}{*}{0.5} & $(40,20,60,60)$ & 0.785734705520629 & 0.785715533029282 & 0.785750736020131 \\
\cline { 3 - 6 } & & $(20,40,60,20)$ & 0.785734705528084 & 0.785714520693756 & 0.785718895194405 \\
\hline \multirow{2}{*}{1.3} & \multirow{2}{*}{1.7} & $(40,60,20,40)$ & 0.785734705594252 & 0.785712470091343 & 0.785725567713847 \\
\cline { 3 - 6 } & & $(60,20,40,60)$ & 0.785734705530414 & 0.785714534388167 & 0.785748611031530 \\
\hline \multirow{2}{*}{1.1} & \multirow{2}{*}{0.9} & $(40,40,40,40)$ & 0.785734705546020 & 0.785713842247919 & 0.785728999913387 \\
\cline { 3 - 6 } & & $(60,60,60,60)$ & 0.785734705546020 & 0.785714339660318 & 0.785729005582190 \\
\hline
\end{tabular}

Table $4: \alpha_{1}=1.5 \quad, \alpha_{1}=1.5 \quad, \beta_{1}=2 \quad, \quad \beta_{2}=2$

\begin{tabular}{|l|l|l|l|l|l|}
\hline $\mathrm{k}$ & $\mathrm{m}$ & $(\mathrm{n} 1, \mathrm{n} 2, \mathrm{~m} 1, \mathrm{~m} 2)$ & $\mathrm{UM}$ & $\mathrm{PT}$ & $\mathrm{MLE}$ \\
\hline 1.5 & \multirow{2}{*}{0.2} & $(20,20,20,20)$ & 0.0000000000416965 & 0.000000001318706 & 0.000000291526563 \\
\cline { 3 - 6 } & & $(60,60,20,40)$ & 0.000000416971124 & 0.000000578485132 & 0.000026615438193 \\
\hline \multirow{2}{*}{1.8} & \multirow{2}{*}{0.5} & $(40,20,60,60)$ & 0.000000416968491 & 0.000000456480257 & 0.000020849454688 \\
\cline { 3 - 6 } & & $(20,40,60,20)$ & 0.000000000416969 & 0.000000001195640 & 0.000000123002043 \\
\hline \multirow{2}{*}{1.3} & \multirow{2}{*}{1.7} & $(40,60,20,40)$ & 0.000000000416971 & 0.000000000680612 & 0.000000045033693 \\
\cline { 3 - 6 } & & $(60,20,40,60)$ & 0.000000416968890 & 0.000000466212429 & 0.000037108268999 \\
\hline \multirow{2}{*}{1.1} & \multirow{2}{*}{0.9} & $(40,40,40,40)$ & 0.000000416969528 & 0.000000576798620 & 0.000054516476000 \\
\cline { 3 - 6 } & & $(60,60,60,60)$ & 0.000000416969528 & 0.000000383695325 & 0.000028051216264 \\
\hline
\end{tabular}

Table $5 \alpha_{1}=3, \quad \alpha_{2}=3, \beta_{1}=1 \quad, \beta_{2}=1, R=0.6250000000$

\begin{tabular}{|l|l|l|l|l|l|}
\hline $\mathrm{k}$ & $\mathrm{m}$ & $(\mathrm{n} 1, \mathrm{n} 2, \mathrm{~m} 1, \mathrm{~m} 2)$ & $\mathrm{UM}$ & $\mathrm{PT}$ & MLE \\
\hline \multirow{2}{*}{1.5} & \multirow{2}{*}{0.2} & $(20,20,20,20)$ & 0.624937468791855 & 0.624999705238945 & 0.624932272202343 \\
\cline { 3 - 6 } & & $(60,60,20,40)$ & 0.624937468537429 & 0.624999441035243 & 0.624953403330766 \\
\hline \multirow{2}{*}{1.8} & \multirow{2}{*}{0.5} & $(40,20,60,60)$ & 0.624937468714946 & 0.625000056038205 & 0.624940516955036 \\
\cline { 3 - 6 } & & $(20,40,60,20)$ & 0.624937469058640 & 0.624999904721153 & 0.624913690218326 \\
\hline \multirow{2}{*}{1.3} & \multirow{2}{*}{1.7} & $(40,60,20,40)$ & 0.624937468534079 & 0.624998720248892 & 0.624943162050123 \\
\cline { 3 - 6 } & & $(60,20,40,60)$ & 0.624937468736565 & 0.624998458153044 & 0.624939686353734 \\
\hline \multirow{2}{*}{0.9} & $(40,40,40,40)$ & 0.624937468734366 & 0.625002127090182 & 0.624942577777575 \\
\cline { 3 - 6 } & & $(60,60,60,60)$ & 0.624937468734366 & 0.625000176762664 & 0.624942314238157 \\
\hline
\end{tabular}


Ibn Al-Haitham Jour. for Pure \& Appl. Sci. 33 (4) 2020

Table 6: $\alpha_{1}=3 \quad, \alpha_{2}=3 \quad, \beta_{1}=1 \quad, \quad \beta_{2}=1$

\begin{tabular}{|l|l|l|l|l|l|}
\hline $\mathrm{k}$ & $\mathrm{m}$ & $\mathrm{n} 1, \mathrm{n} 2, \mathrm{~m} 1, \mathrm{~m} 2)$ & $\mathrm{UM}$ & $\mathrm{PT}$ & $\mathrm{MLE}$ \\
\hline 1.5 & \multirow{2}{*}{0.2} & $(20,20,20,20)$ & 0.000000003910152 & 0.0000000003234843 & 0.000000239325138 \\
\cline { 3 - 6 } & & $(60,60,20,40)$ & 0.000000003910184 & 0.0000000001870957 & 0.0000000085454692 \\
\hline \multirow{2}{*}{1.8} & \multirow{2}{*}{0.5} & $(40,20,60,60)$ & 0.000000003910162 & 0.0000000001122373 & 0.000000045087534 \\
\cline { 3 - 6 } & & $(20,40,60,20)$ & 0.000000003910119 & 0.000000002522772 & 0.000000181399978 \\
\hline \multirow{2}{*}{1.3} & \multirow{2}{*}{0.7} & $(40,60,20,40)$ & 0.000000003910184 & 0.000000001857193 & 0.000000189712498 \\
\cline { 3 - 6 } & & $(60,20,40,60)$ & 0.000000003910159 & 0.000000001040114 & 0.000000030728521 \\
\hline \multirow{2}{*}{1.1} & \multirow{2}{*}{0.9} & $(40,40,40,40)$ & 0.000000003910159 & 0.000000001408268 & 0.000000056503599 \\
\cline { 3 - 6 } & & $(60,60,60,60$ & 0.000000003910159 & 0.000000000964106 & 0.000000038498170 \\
\hline
\end{tabular}

\section{Conclusion}

the cascade reliability model (2+1) $R=p\left[X_{1} \geq Y_{1}, X_{2} \geq Y_{3}\right]+p\left[X_{1}<\right.$ $\left.Y_{1}, X_{2} \geq Y_{2}, X_{3} \geq Y_{3}\right]+p\left[X_{1} \geq Y_{1}, X_{2}<Y_{2}, X_{3} \geq Y_{3}\right] \quad$ based on inverted exponential distribution were used in this paper to verify the performance of different estimators which are ; Maximum likelihood estimation, Unbiased estimation method and Preliminary test single stage shrinkage (PTSSS) estimator using different samples $(20,40$, and 60). The Monte Carlo Simulation was exhibited to analyses and comparison between these methods. the results show that the performance of (PTSSS) estimator was appropriate behavior and it is efficient estimator than the others in the sense of $M S E$ at most. While $\widehat{R}_{(U M V U)}$ had the second rank and followed by $\widehat{R}_{S S S}$.

\section{References}

1. Pandit, S. N. N.; Sriwastav, G. L. Studies in Cascade Reliability I. IEEE Trans. on Reliability.1975, 24,1, 53-56.

2. Rekha, A.; Raju, V.C. C. Cascade System Reliability with Rayleigh Distribution. Botswana Journal of Technology.1999, 8, 14-19.

3. Sundar, T. S. Case Study of Cascade Reliability with Rayleigh Distribution. International Journal of Computer Technology and Electronics Engineering. 2012, 2, 3, 78-87.

4. Devi, M.T. Cascade System Reliability with Stress and Strength Follow Lindley Distribution. International Journal of Engineering Research \& Technology. 2016, 5, 09,708-714

5. Dong, H.; Cui, L. System Reliability under Cascading Failure Models. IEEE Transactions on Reliability. 2015, 65, 2,1-12.

6. Doloi, C.; Gogoi, J. A cascade Reliability Model For Exponential and Lindley Distributions. International Journal of Advanced Research. 2017, 5, 4, 43-54.

7. Reddy, D. Cascade and system Reliability for exponential distributions. DJ Journal of Engineering and Applied Mathematics. 2016, 2, 2, 1-8.

8. Kanaparthi, R.; Palakurthi, J. ; Sanka, L.N.G.D. Cascade and System Reliability for the New Rayleigh-Pareto Distribution. International Journal of Science and Research. 2019, 8, 2, 1865- 1867. 
9. Salman, A.S.; Ali, A.H; Salman, M.D. Preliminary Test Single Stage Shrinkage Estimator for the Scale Parameter of Gamma Distribution. American Journal of Mathematics and Statistics. 2014, 4, 3, 131-136.

10. Salman, A.S., Hadi, R.A. Preliminary Test Shrinkage Estimators for The Shape Parameter of Generalized Exponential Distribution. International Journal of Applied Mathematical Research.2016, 5, 4, 162-166.

11. Salman, A.S.; Hamad, A. M.; Abdul-Nabi, A. I. Pretest Single Stage Shrinkage Estimator for the Shape Parameter of the Power Function Distribution. International Journal of Mathematics Trends and Technology. 2016, 63, 1, 56-59.

12. Salman, A.S.; Hassoun, I.O. An Efficient Single Stage Shrinkage Estimator for the Scale parameter of Inverted Gamma Distribution. Ibn Al-Haitham J. for Pure \& Appl. Sci. 2017, 30 ,1 , 169-176.

13. Killer, A. Z. ; Kamath, A. R. Reliability analysis of CNC machine tools. Reliability Engineering. 1982, 3, 449-473.

14. Tawfiq, LNM; Oraibi. YA. Fast Training Algorithms for Feed Forward Neural Networks. Ibn Al-Haitham Journal for Pure and Applied Science. 2017, 26, 1: 12751280 .

15. Singh, S.K.; Singh, U.; Kumar, D. Bayes estimators of the reliability function and parameter of inverted exponential distribution using informative and non-informative priors. Journal of Statistical Computation and Simulation.2013, 83,12, 2258-2269.

16. Oguntunde, P.E.; Adejumo, A.; Balogun, O.S. Statistical properties of the exponentiated generalized inverted exponential distribution. Applied Mathematics. 2014, 4, 2, 47-55.

17. Singh, S.K; Singh, U.;Yaday,A,;Viswkarma, P.K. On the estimation of stress strength reliability parameter of inverted exponential distribution. International Journal of Scientific World, 2015, 3, 1, 98-112.

18. Devor, J.L.; Berk, K.N. Modern Mathematical Statistics with Applications. 2012, Second edition, Springer Texts in Statistics. 